\title{
Development and validation of measure of household food insecurity in Northern Rao PDR
}

\author{
Ji-Yun Hwang ${ }^{1}$, Seoungwoo John Lee ${ }^{1}$ and Mi Nae Yang ${ }^{2}$ \\ ${ }^{1}$ Department of Food service management and Nutrition, Sangmyung University, Seoul, Republic of Korea and \\ ${ }^{2}$ Nutrition Education Major, Graduate School of Education, Seoul, Korea, Republic of
}

\section{Abstract}

Introduction: Lao PDR is one of the poorest countries in the world, ranked the 138th of 188 developing countries. Although household food insecurity has been prevalent in Northern Rao PDR, no valid measurement of household food insecurity exists. Therefore, the study was to develop a household food insecurity questionnaire and verify the validity of the questionnaire in the rural areas of Lao PDR.

Materials and Methods: Using a multistage-sampling cross-sectional study, the sample included 373 households with children under 5 years of age living in Xieng Khouang, Lao PDR. The questionnaire was composed of 18 items based on the US household Food Security Survey Module and partially modified to local circumstances. Internal consistency of questions measured with Cronbach's $\alpha$ was adequate, result of 0.746 . External validity was evaluated through comparison analyses between food insecurity levels and household socioeconomic indicators.

Results and discussion: Among 373 households, 42.7\% were food-insecure and 4.3\% were food-insecure with hunger. There were more food-insecure households in those with lower maternal education levels $(\mathrm{P}<0.001)$, greater number of household members $(\mathrm{P}<$ 0.001), longer travel time to farmland $(\mathrm{P}=0.003)$, and lower household asset scores $(\mathrm{P}<0.001)$. Households with the residence made of natural material for walls $(\mathrm{P}<0.001)$, roofs $(\mathrm{P}<0.001)$ and floors $(\mathrm{P}<0.001)$ as well as without electricity $(\mathrm{P}<0.001)$, pipe to supply drinking water $(\mathrm{P}<0.001)$, and income other than agriculture $(\mathrm{P}<0.001)$ had higher food insecurity than their counterparts. In addition, inadequate maternal prenatal care and education, child health care, and vaccinations increased household food insecurity. Mothers and children in food-insecure households had lower intakes of various food groups including protein-source foods. This study would provide a valid instrument to assess food insecurity and be used in the future as an evaluation tool for the improvement of nutritional deficiencies as well as a tool for selection of target subjects in Northern Rao PDR.

\section{Acknowledgment}

This study was supported by the grant from National Research Foundation of Korea (NRF-2017R1A2B1011821).

\section{Conflict of Interest}

There is no conflict of interest. 